\title{
INFLUÊNCIA DO CONDICIONAMENTO ÁCIDO NO REPARO DE CERÂMICAS ODONTOLÓGICAS UTILIZANDO RESINA COMPOSTA
}

\author{
Francisca Carvalho Souza \\ Lorena Rodrigues Santos
}

Anderson Pinheiro De Freitas

Silvio Alberto Almeida Silveira

\begin{abstract}
Marcio Vieira Lisboa
\end{abstract}
José Augusto Ataíde Lisboa
Graduada na Universidade Federal da Bahia.

Graduanda no curso de Odontologia pela Universidade Federal da Bahia.

Doutorado em Reabilitação Oral na University of Western Ontário. Doutorado em Materiais Dentários pela Universidade de São Paulo e mestrado em Materiais Dentários pela Faculdade de Odontologia de Bauru. Professor Adjunto da Universidade Federal da Bahia.

Especialista em Gestão de Empresas e em Metodologia da Educação. Mestrado em gestão empresarial. Funcionário Público Federal da Faculdade de Odontologia da Universidade Federal da Bahia.

Mestrado em Odontologia pela Universidade Federal da Bahia e doutorado em Clínica Odontológica Prótese Dental pela Universidade Estadual de Campinas. Professor adjunto da Universidade Federal da Bahia

Mestrado em Odontologia (Prótese Dentária) pela Universidade de São Paulo e doutorado em Odontologia pela Faculdade de Odontologia da Universidade Federal da Bahia (UFBA). Professor adjunto da UFBA.

Endereço para correspondência: lorenarodrigues@ hotmail.com

\section{Resumo}

O uso das restaurações cerâmicas é constantemente indicado e utilizado nos consultórios odontológicos devido as suas qualidades estéticas e estruturais, no entanto mesmo sendo um material com excelentes propriedades mecânicas podem apresentar fraturas. A substituição total da peça protética tem custo elevado, e necessita de bastante tempo clínico. Assim, o reparo das cerâmicas utilizando resina composta fotopolimerizável tem sido uma boa alternativa em casos de fratura. O objetivo desse trabalho é avaliar, por meio de uma revisão de literatura, a influência do condicionamento ácido no reparo de cerâmicas com resina composta. A coleta de dados foi realizada através da seleção de artigos publicados nas bases de dados Scielo, LILACS e Bireme, utilizando os seguintes descritores: tratamento de superfície, resina composta e cerâmicas odontológicas. De acordo com os achados pesquisados, o reparo da superfície cerâmica fraturada pode ser realizado intra-oralmente através do tratamento mecânico e químico da superfície fraturada. Para cerâmicas ácido sensíveis é relatado na literatura a efetividade do condicionamento com ácido fluorídrico na promoção de alteração superficial, no entanto, a longevidade desta adesão entre cerâmica e resina composta após o condicionamento ácido ainda é questionável.

Palavras-chave: Cerâmica; Resistência à tração; Ácido fluorídrico. 


\title{
INFLUENCE OF ACID ETCHING IN THE REPAIR OF DENTAL CERAMICS USING COMPOSITE RESIN
}

\begin{abstract}
The use of ceramic restorations is constantly indicated and used in dental clinics because of their structural and aesthetic qualities, however despite being a material with excellent mechanical properties it may present fractures. The complete replacement of a prosthetic restoration has an elevated cost and needs a longer clinical time. So the repair of ceramics using composite resin has been a good alternative in cases of fracture. The objective of this study is to evaluate through a literature review the influence of acid etching in the repair of ceramic with composite resin. The data collection was performed through the selection of articles published in the databases SciELO, LILACS and Bireme using the following descriptors: surface treatment, composite resin and dental ceramics. According to the findings gathered, the repair of fractured ceramic surface can be performed intra-orally through the mechanical and chemical treatment of the fractured surface. For acid sensitive ceramics it is reported in literature the effectiveness of hydrofluoric acid treatment in promoting superficial alterations, however, the longevity of such adhesion between ceramic and composite resin after etching is still questionable.
\end{abstract}

Keywords: Ceramic; Tensile strength; Hydrofluoric acid.

\section{INTRODUÇÃO}

Nos últimos anos, com a crescente busca por estética, as cerâmicas odontológicas vêm sendo cada vez mais utilizadas, pois constituem o material que melhor reproduz as propriedades ópticas do esmalte e dentina, como fluorescência, opalescência e translucidez e tem sido, atualmente, a principal alternativa de tratamento restaurador indireto para a estrutura dental. ${ }^{(1)}$ Em contra partida às vantagens estéticas deste material, existem características preocupantes, como a friabilidade e a baixa resistência à tração. Buscando minimizar essas deficiências, as pesquisas visam a obtenção de cerâmicas mais resistentes e duráveis através da incorporação de vidros cerâmicos e adição de cristais para reforço, como o quartzo e a alumina. ${ }^{(2)}$

A contínua evolução dos sistemas adesivos tem possibilitado aumento significativo das alternativas restauradoras. Aliado a esse fato, os materiais restauradores, em particular as cerâmicas, também experimentaram melhora considerável em suas propriedades mecânicas, ópticas e características de manipulação. ${ }^{(3)}$ Atualmente, têm-se disponível no mercado, sistemas cerâmicos que apresentam melhor resistência a fratura em relação às cerâmicas feldspáticas, estando entre eles: Sistema cerâmico infiltrado por vidro (Sistema In-Ceram Spinel, Zircônia e Alumina), Sistemas cerâmicos prensados (Sistema IPS E.Max, Empress I e II) e Sistemas cerâmicos fresados (Procera AllCeram e cerâmicas à base de zircônia tetragonal policristalina estabilizada com ítria - Y-TZP). ${ }^{(4)}$ 
Apesar das características mecânicas desfavoráveis das cerâmicas feldspáticas, Ozcan ${ }^{(5)}$ relata em seu estudo uma prevalência de 5\% a 10\% de falhas após 10 anos de uso. Segundo Raposo, Neiva, Silva, Carlo, Mota e Prado ${ }^{(6)}$ essas falhas são decorrentes do trauma e da fadiga que os materiais dentários e as interfaces adesivas estão submetidas ao estresse no ambiente bucal. ${ }^{(6,7,8)}$ As falhas podem ser fratura da cerâmica ou destruição da ligação cerâmica-metal, e tal ocorrência gera um desconforto estético, funcional e econômico, tanto para o profissional como para o paciente. ${ }^{(5)}$

No entanto, a substituição total da restauração fraturada nem sempre é viável do ponto de vista econômico, estético ou biológico. ${ }^{(7)} \mathrm{O}$ reparo intraoral de cerâmicas com resina composta oferece aos pacientes uma alternativa viável para a substituição complete da prótese defeituosa, podendo aumentar a longevidade clínica das restaurações. ${ }^{(9)}$ Haneda, Almeida-Junior, Fonseca e Adabo $^{(7)}$ e Ozcan, Valandro, Amaral, Leite e Bottino ${ }^{(10)}$ afirmam que o reparo é uma solução possível para os casos em que a restauração fraturada apresenta adaptação satisfatória e integridade periodontal. O reparo da superfície cerâmica fraturada pode ser realizado intraoralmente com auxílio de resina composta fotopolimerizável através do tratamento mecânico e químico da superfície fraturada. ${ }^{(7)} \mathrm{O}$ sucesso e a longevidade do reparo efetuado é dependente da união cerâmica/resina, ${ }^{(11,12)}$ e está diretamente relacionado a fatores que envolvem o tratamento da superfície da cerâmica como: uso de jateamento com partículas de óxido de alumínio ou asperização por pontas diamantadas, condicionamento químico da superfície da cerâmica, tipo e concentração de ácido utilizado no condicionamento e o tempo de aplicação. ${ }^{(11)}$

Muitos estudos são publicados relatando a eficiência e a importância do tratamento da superfície das restaurações indiretas em diferentes cerâmicas. ${ }^{(13)} \mathrm{O}$ tratamento de superfície tradicionalmente empregado consiste na utilização de ácido fluorídrico em concentrações que variam de 2 a 10\%, em períodos que variam de 1 a 4 minutos de acordo com cada fabricante, seguido da aplicação de agente de união silano. ${ }^{(2)}$

Este trabalho pretende abordar através de uma revisão de literatura a influência do condicionamento ácido no reparo intra-oral de restaurações em cerâmica pura utilizando resinas composta. 


\section{METODOLOGIA}

A coleta de dados foi realizada através de uma busca nos bancos de dados da Bireme, MEDLINE (PubMed) e Scielo, para artigos publicados na literatura entre os anos de 2000 e 2012. Não foi selecionado nenhum filtro para refinar a pesquisa nem foi aplicada restrição de idioma. As palavras chaves utilizadas foram: "tratamento de superfície", "resina composta", "cerâmicas de revestimento". Foram coletados 40 artigos, dos quais 31 foram selecionados por se enquadrar no tema proposto.

A elegibilidade dos artigos foi determinada pela leitura do resumo e foi conduzida de forma independente pelos pesquisadores. A avaliação da metodológica e da extração de dados das publicações incluídas foram previamente estabelecidas pelos revisores.

\section{REVISÃO DE LITERATURA}

Em restaurações cerâmicas, quando ocorre fratura do revestimento estético da cerâmica, a possibilidade de reparo cerâmico através de resinas compostas é uma técnica aplicável, que pode restaurar a estética e função do paciente. ${ }^{(5)}$ As vantagens do reparo, comparadas à total substituição da prótese, seriam minimizar a perda de estrutura sadia, preservando o remanescente dentário e promover maior longevidade da restauração a um menor custo operacional. ${ }^{(14)}$ Em que pese às vantagens inerentes ao reparo com resina composta da cerâmica, a infiltração marginal na interface destes materiais interfere na longevidade desta conduta, neste caso não é indicado o reparo. $^{(15)}$

Segundo Zavanelli, Dekon, Silva, Pesqueira, Costa e Takeshita, ${ }^{(16)}$ as falhas em Prótese Parcial Fixa podem ser divididas em três grupos: falhas de origem biológica, falhas de origem mecânica e associação mecânico-biológica. Quando não são consideradas causas biológicas como doença cárie e envolvimento periodontal, o maior problema mecânico envolvido na longevidade destas restaurações são falhas estruturais da cerâmica, predispostas por fatores como indicação incorreta do material para confecção da prótese, preparo dental insuficiente e/ou irregular, procedimentos laboratoriais inadequados. No entanto, reparo só é indicado para os casos em que a restauração fraturada apresenta satisfatória adaptação e integridade periodontal. ${ }^{(7)}$ 
Segundo Raposo et al. ${ }^{(6)}$ alguns fatores que indicam o reparo da restauração cerâmica são o alto custo para substituição total da prótese, possível trauma que pode causar no dente restaurado, dificuldade de remoção das restaurações e demanda de pacientes para uma resolução emergencial do caso. A análise da viabilidade da realização do reparo e a eleição deste tratamento devem ser fundamentadas nas condições clínicas apresentadas e depende da localização, extensão e do tipo da falha ocorrida, da qualidade do material empregado, na expectativa da longevidade do reparo e no custo envolvido.

Estruturalmente, as cerâmicas odontológicas se caracterizam por possuir uma fase cristalina circundada por uma fase vítrea. Dependendo da natureza e da quantidade da fase cristalina presente, as propriedades mecânicas e ópticas variam amplamente, assim pode diferir o efeito do condicionamento com ácido fluorídrico sobre a superfície. ${ }^{(17,18)}$

\subsection{Cerâmicas Feldspáticas}

Apesar das excelentes propriedades mecânicas das cerâmicas feldspáticas, estas são materiais friáveis que podem apresentar fraturas internas devido a esforços de tração, fadiga, compressão ou falhas espontâneas, em função de defeitos na superfície, ${ }^{(2,7,11,19,20)}$ traumas, ajuste oclusal inadequado, hábitos parafuncionais, ${ }^{(20)}$ a falha da união adesiva, ${ }^{(6)}$ porosidades na cerâmica, ${ }^{(5)}$ redução inadequada da preparação dentária, ${ }^{(7)}$ mudanças de temperaturas, saliva e $\mathrm{pH} .{ }^{(6)}$

O condicionamento com ácido fluorídrico dissolve os componentes vítreos e cristalinos deste tipo de cerâmica, alterando de forma significativa sua morfologia superficial, promovendo irregularidades representadas por microporos retentivos, fendas e sulcos. ${ }^{(12,21,22)}$ Dessa forma, têm a propriedade de aumentar a molhabilidade do cimento na superfície. ${ }^{(23)}$

Zavanelli et al. ${ }^{(16)}$ mostram que a corrosão da superfície cerâmica ácido sensível é provocada pela ação dos íons fluoreto sobre a malha de silício-oxigênio, potencializando este efeito pela diminuição do $\mathrm{pH}$ e aumento da concentração do íon fluoreto no ácido utilizado. Os íons fluoreto em ambiente ácido atacam os componentes vítreos das cerâmicas formando fluorsilicato solúvel em água, tornando assim a superfície cerâmica irregular e alterando suas propriedades físicas e estéticas. 
A resistência de união de uma resina composta a um substrato cerâmico é tradicionalmente baseada em mecanismos de retenção micromecânica e adesão química via organossilanos. ${ }^{(22)} \mathrm{O}$ uso do ácido fluorídrico é o tratamento de superfície de cerâmica mais utilizados no reparo de restaurações cerâmicas ácido-sensíveis. Este procedimento, seguido da aplicação de silano, produz união clinicamente aceitável da resina composta para cerâmica à base de sílica. ${ }^{(12,22)}$

As alterações na superfície da cerâmica são observadas principalmente após a exposição ao ácido fluorídrico durante 2 minutos, o que provoca mudanças na translucidez e acabamento superficial. Assim, pode-se inferir que a duração da exposição do ácido selecionado é um importante fator a ser considerado nos efeitos sobre o material, e deve ser escolhido com critério para não comprometer as propriedades desse material, obtendo-se apenas o efeito desejado. ${ }^{(16)}$

Raposo et al., ${ }^{(6)}$ afirmam em seu trabalho que uma adequada adesão entre cerâmicas e resinas compostas é conseguida com a aplicação de agente de união silano e adesivo. A silanização promove a adesão química do material restaurador adesivo com a cerâmica. Esta adesão ocorre por meio do seguinte mecanismo: o silano é uma molécula bifuncional, ${ }^{(7)}$ sendo que cada extremidade de sua molécula reage com diferentes superfícies, uma inorgânica da cerâmica, e a outra orgânica, da resina composta, ocorrendo então a formação de uma ligação covalente entre ambas. ${ }^{(6)}$ Essa tecnologia melhora a adesão química do reparo de cerâmicas com resinas compostas. Também pode melhorar o molhamento da superfície cerâmica pelo adesivo, pois a superfície com revestimento de silano é organofílicas para o adesivo. ${ }^{(12)}$

Autores afirmam que o reparo da cerâmica deve ser realizado pelos seguintes passos: prévia asperização da mesma (de preferência um jateamento), condicionamento com ácido fluorídrico, aplicação de um agente para união química (silano) e então sistema adesivo e a resina composta. ${ }^{(10,14,20,24-26)}$ A opção pela asperização com instrumento cortante rotatório diamantado deve-se em virtude da realidade clínica. Este instrumento, além de ter a capacidade de criar irregularidades na superfície da cerâmica para uma posterior retenção mecânica, está presente em qualquer consultório odontológico. O jateamento promove a criação de áreas microrretentivas na superfície da cerâmica, porém exige um equipamento específico, o que dificulta sua utilização. ${ }^{(10)}$ 


\subsection{Sistema cerâmico infiltrado por vidro - Sistema InCeram}

A cerâmica In-Ceram foi desenvolvida visando melhorar os problemas relacionados com a resistência a fratura e tenacidade. Sua composição consiste em duas fases tridimensionais interpenetradas: uma fase de alumina (óxido de alumínio) e uma fase vítrea (à base de óxido de lantânio), sendo sua confecção baseada em alumina porosa que, posteriormente, é infiltrada por vidro. Este sistema apresenta três variedades, de acordo com o seu principal componente: alumina $\left(\mathrm{Al}_{2} \mathrm{O}_{3}\right)$ - In-Ceram Alumina, spinel $\left(\mathrm{MgAl}_{2} \mathrm{O}_{4}\right)$ - In-Ceram Spinel e zircônia $\left(\mathrm{Al}_{2} \mathrm{O}_{3} \mathrm{ZrO}_{2}\right)$ - In-Ceram Zircônia. ${ }^{(4)}$

O conhecimento do tipo de cerâmica empregado pode ser importante para a escolha entre a concentração do ácido e seu tempo de aplicação. Entretanto, para cerâmicas do sistema InCeram, em face das suas características estruturais, o condicionamento ácido não é indicado pois a infra-estrutura de alumina infiltrada de vidro não sofre ação do mesmo. ${ }^{(2)}$

\subsection{Sistemas cerâmicos prensados - Sistema IPS e.max, Empress}

O sistema IPS e.max ou Empress é baseado em cerâmica vítrea reforçada por cristais de leucita (35-55\% - Sistema IPS Empress I ) ou reforçada por cristais de dissilicato de lítio (6065\% - Sistema IPS emax e Empress II). Esse sistema simplificou o problema de contração durante a queima da cerâmica, comum para as feldspáticas, devido à alta pressão de injeção da cerâmica no molde em alta temperatura. ${ }^{(27)}$

Della Bona et al., ${ }^{(12)}$ avaliaram o modo de fratura de cerâmicas com leucita (IPS Empress) e dissilicato de lítio (IPS Empress 2), tratadas com diferentes ácidos e tempos de aplicação, e verificaram que o condicionamento com ácido fluorídrico 9,5\%, associado ao agente silano, produziu valores mais altos de resistência à fratura e que a ação deste ácido foi mais significante no IPS Empress 1, provavelmente pela presença da sílica na fase vítrea desta cerâmica.

Jorge $^{(28)}$ em seu trabalho analisou a rugosidade de superfície e constatou que o ácido fluorídrico possui a capacidade de condicionar quimicamente alguns tipos de cerâmicas, atuando diretamente na porção vítrea e, no caso do IPS Empress 2, expondo os cristais de dissilicato de 
lítio, gerando irregularidades na superfície do material, o que explica o aumento da rugosidade encontrado no grupo das cerâmicas previamente polidas.

Os tratamentos de superfície de cerâmicas feldspáticas e a base de leucita tem sido extensivamente avaliados, comprovando a efetividade do acido fluorídrico. ${ }^{(2)}$ Todavia, segundo Aras e León ${ }^{(29)}$ para cerâmicas a base de dissilicato de litio e a base de alumina, tal procedimento não está bem esclarecido.

\subsection{Sistemas cerâmicos fresados}

Cerâmica à base de zircônia tetragonal policristalina contendo ítrio (Y-TZP):

O óxido de ítrio é adicionado na zircônia pura com o objetivo de estabilizar a fase cúbica ou tetragonal na temperatura ambiente, gerando um material polifásico conhecido como zircônia estabilizada. A estabilização na fase tetragonal é responsável pela alta tenacidade à fratura da YTZP. A Y-TZP não possui fases vítreas devido à microestrutura policristalina, o que evita o fenômeno de sua degradação ou desestabilização pela saliva e conseqüente aumento da propagação de trincas. ${ }^{(30)}$

Cavalcanti, Foxton, Watson, Oliveira, Gianni e Marchi ${ }^{(13)}$ cita no seu estudo que o condicionamento ácido não é eficiente para as cerâmicas de zircônia tetragonal policristalina contendo ítrio (Y-YZP), devido à sua falta de sílica e de vidro. Na avaliação das imagens em microscopia após o tratamento de superfície das cerâmicas concluiu-se que a abrasão por jateamento de partículas com $\mathrm{Al}_{2} \mathrm{O}_{3}$ parece ser um método mais eficiente para modificar superfícies da zircônia em comparação com irradiação com laser. Mesmo embora alguns fabricantes de Y-TZP sugerirem o uso de abrasivos ou revestimento adesivo antes de cimentação, o efeito dos tratamentos de superfície sobre as propriedades mecânicas dos materiais Y-TZP é controversa. Portanto, tratamento de superfície adequado para a Y-TZP cerâmica ainda tem de ser determinada.

A cerâmica com alto conteúdo de alúmina (Sistema Procera / 99,9\% de $\mathrm{Al}_{2} \mathrm{O}_{3}$ ) se apresenta densamente sinterizada e utiliza a tecnologia CAD/CAM. Esse sistema apresenta características mecânicas diferenciadas em relação aos demais sistemas citados anteriormente, mas ainda há certa insegurança quanto sua cimentação adesiva. ${ }^{(4)}$ 
Sintetizando, para as cerâmicas feldspáticas e para as reforçadas com dissilicato de lítio e leucita, o condicionamento da superfície com ácido fluorídrico e o jateamento da superfície com óxido de alumínio são meios eficazes na promoção de união mecânica a resina composta e a silanização é um método que proporciona a união química entre a fase inorgânica da cerâmica e a fase orgânica do material resinoso aplicado sobre a superfície cerâmica condicionada. Já para as cerâmicas que apresentam como componente principal os óxidos de alumínio e zircônia (InCeram, Procera, Y-TZP), o condicionamento com ácido fluorídrico não se mostra eficiente, devido a ausência da fase vítrea e redução do conteúdo de sílica. Assim, podem ser utilizados métodos alternativos para proporcionar uma adequada união desses materiais, como o jateamento com óxido de alumínio.

Os passos clínicos e/ou fatores mencionados no presente artigo não são consenso na literatura, além disso, algumas limitações devem ser salientadas, em especial sobre a resina composta, como a redução da estabilidade de cor e resistência ao desgaste em relação à cerâmica. Por isso, em casos de reparo em cerâmica com resina composta é importante realizar um posterior acompanhamento do caso com reavaliações periódicas.

\section{DISCUSSÃO}

A revisão de literatura mostrou a grande busca pelo aprimoramento na adesão entre restauração de cerâmica e resina composta. Considerando a possibilidade de reparo em falhas cerâmicas, a adesão resina/cerâmica representa benefícios para o profissional e paciente como menor tempo clínico, facilidade da técnica, menor custo, minimização da perda de estruturas sadias, bons resultados estéticos e funcionais.

A resolução clínica de intercorrências relacionadas a fratura em restaurações cerâmicas pela técnica de reparo com resina composta deixa, contudo, algumas dúvidas em relação à resistência adesiva entre a resina e o substrato reparado e ainda, se ela elevaria efetivamente a longevidade da restauração.

Campos, Telles, Galhano, Camargo, Valandro e Mallmann ${ }^{(2)}$ afirmam que o tratamento de superfície preferencial para as cerâmicas com sílica (vítreas ou feldspáticas) é o condicionamento com ácido fluorídrico (2,5-10\%) durante 2 a 3 minutos com posterior aplicação 
de um agente silano. Em concordância em relação ao uso do ácido fluorídrico e do silano, Murad $^{(14)}$ relataram em seu estudo que a condição de superfície afeta significantemente a resistência adesiva da resina composta aderida a esta superfície. O adequado preparo da superfície consiste em: desgaste físico da mesma, atuação de substância ácida e aplicação de adesivos, além do uso do silano.

Jorge $^{(28)}$ em seu trabalho analisaram a rugosidade de superfície e constataram que o ácido fluorídrico possui a capacidade de condicionar quimicamente alguns tipos de cerâmicas, atuando diretamente na porção vítrea, mostrando assim sua efetividade. Corroborando com esse estudo, Raposo et al. ${ }^{(6)}$ e Aras e León ${ }^{(29)}$ afirmam que o procedimento de reparo para cerâmicas feldspáticas requer o condicionamento da sua superfície com acido fluorídrico prévio, pois este tratamento tem sido extensivamente avaliado, com comprovada efetividade.

$\mathrm{Na}$ aplicação do ácido fluorídrico $9,5 \%$ durante 2 minutos, pode-se observar condicionamento mais severo do que de outros ácidos ou diferentes tempos de exposição, porém avaliando a resistência ao cisalhamento, verificando que não houve diferença entre os tratamentos empregados. $^{(16)}$ Desta mesma forma Garone, Russo e Netto ${ }^{(20)}$ chegaram a um resultado semelhante ao avaliar a união entre resina composta e diferentes tipos de cerâmicas tratadas com ácido fluorídrico (HF). O grupo de cerâmica feldspática (Noritake) foi dividido em 3 grupos, um grupo tratado com ácido fluorídrico a $10 \%$ durante 2 minutos, outro grupo com HF a $5 \%$ durante 1 minuto e o terceiro grupo com HF 5\% durante 20 segundos. Estes não apresentaram diferenças estatísticas entre si.

Contrário a maioria dos trabalhos presentes na literatura, Spohr, Sobrinho, Cansani, Sinhoret e Knowles ${ }^{(31)}$ afirmam que o condicionamento ácido da cerâmica pode ser eliminado, resultando em uma redução no tempo de operação e eliminação do perigo de armazenar ácido flourídrico. Apenas a aplicação de silano daria resistência de união comparável com condicionamento ácido e supera a própria força de coesão da cerâmica.

Kussano, Bonfante, Batista e Pinto ${ }^{(8)}$ em seu estudo sobre a avaliação da adesão entre resina composta e cerâmica, relatam que o silano proporciona um reparo estável da superfície fraturada. Afirmam também que não é necessária utilizar o ácido fluorídrico para obter boa adesão entre compósito e cerâmica. O agente silano parece ser o componente essencial para um procedimento de reparo, modificando a estrutura de superfície, tornando-a mais reativa e 
permitindo adesão da cerâmica com o compósito em ligações inorgânicas e orgânicas. Os autores observaram que a força de união entre resina composta/cerâmica era relativamente fraca quando não se utilizava o silano, independentemente do tipo de tratamento de superfície, com a ocorrência da falha na interface.

Nesta discussão, observa-se que o mecanismo envolvido em reparos de cerâmicas com resina composta é multifatorial, e os métodos de avaliação fornecem dados parciais. A cavidade bucal é um meio ambiente bastante complexo, sendo difícil reproduzir todas as suas variáveis em um único estudo, no entanto, há uma predileção de diversos autores pelo uso do ácido fluorídrico associado ao silano ${ }^{(2,6,16,20,29,28)}$ e de outro grupo de autores pelo uso apenas de silano, por considerarem que a rugosidade produzida pelo ácido fluorídrico é dispensável para a adesão. ${ }^{(8,31)}$

\section{REFERÊNCIAS}

1. Guerra CMF, Neves CAF, Almeida ECB, Valones MAA, Guimarães RP. Estágio atual das cerâmicas odontológicas. Int J Dent 2007;6(3):90-95.

2. Campos L, Telles M, Galhano GA, Camargo FP, Valandro LF, Mallmann A. Efeito do tempo de condicionamento da superfície cerâmica sobre a resistência adesiva entre uma cerâmica de fluorapatita e um cimento resinoso. Cienc. odontol. bras. 2005;8(3):71-76.

3. Conceição EN. Dentística saúde e estética. Porto Alegre: Artmed; 2000. p. 249-262.

4. Gomes EA, Assunção WG, Rocha EP, Santos PH. Cerâmicas odontológicas: o estado atual. Cerâmica 2008;(54):319-325.

5. Özcan, M. Evaluation of alternative intra-oral techniques for fractured ceramic-fused-to-metal restorations. J Oral Rehabil 2003;30(2):194-203.

6. Raposo LHA, Neiva NA, Silva GR, Carlo HL, Mota AS, Prado CJ, et al . Ceramic restoration repair: report of cases. J Appl Oral Sci. 2009;17(2):140-144.

7. Haneda IG, Almeida-Junior AA, Fonseca RG, Adabo GL. Intraoral repair in metal-ceram ic prostheses: a clinical report. Rev. odontol. Univ. Cid. São Paulo 2009; 21(3):282-287.

8. Kussano CM, Bonfante G, Batista JG, Pinto JHN. Evaluation of Shear Bond Strength of Composite to Porcelain According to Surface Treatment. Braz Dent J 2003; 14(2):132-135.

9. Khoroushi M, Motamedi SH. Shear Bond Strength or Composite-Resin too Porcelain: Effect of Thermocycling. Jornal of Dentistry. 2007;4(1):21-26. 
10. Özcan M, Valandro LF, Amaral R, Leite F, Bottino MA. Bond strength durability of a resin composite on a reinforced ceramic using various repair systems. Dent mater 2009;25:1477-1483.

11. Melo RM, Valandro LF, Bottino MA. Microtensile Bond Strength of a Repair Composite to Leucite-Reinforced Feldspathic Ceramic. Braz Dent J 2007;18(4):314-319.

12. Della Bona A. Caracterizing ceramics and the interfacial adhesion to resin: II-The relationship of surface treatment, bond strength, interfacial toughness and fractography. J Appl Oral Sci 2005;13(2):101-109.

13. Cavalcanti AN, Foxton RM, Watson TF, Oliveira MT, Gianni M, Marchi GM. Bond Strength of Resin Cements to a Zirconia Ceramic with Different Surface Treatments. Oper Dent 2009;34(3):280-287.

14. Murad CG. Avaliação da resistência à tração de reparos em resina composta. Bauru. [dissertação]. Bauru: Faculdade de Odontologia de Bauru, Universidade de São Paulo; 2003.

15. Mello EB, Silva RCS. Influência do tratamento de superfície na infiltração marginal de reparos em porcelana. J Bras Clin Odontol Int. 2002;6(36):451-455.

16. Zavanelli AC, Dekon SFC, Silva CR, Pesqueira AA, Costa OS, Takeshita EM. Efeito dos tratamentos superficiais para reparo em cerâmica: avaliação por meio da microscopia eletrônica de varredura. Cienc. odontol. bras. 2006;9(3):66-72.

17. Muzilli M. Influência do tratamento de superfície na rugosidade Ra e dureza Vickers de cerâmicas para cobertura incisal. Piracicaba. [dissertação]. Piracicaba: Faculdade de Odontologia de Piracicaba, Universidade Estadual de Campinas; 2005.

18. Butze JP. Avaliação da topografia superficial de cerâmicas submetidas a diferentes tratamentos de superfície. [trabalho de conclusão de curso]. Porto Alegre: Pontifícia Universidade Católica do Rio Grande do Sul; 2009.

19. Vasquez IMT. Avaliação In Vitro da resistência ao cisalhamento de três agentes cimentantes em porcelana feldspática. Bauru. [dissertação]. Bauru: Faculdade de Odontologia de Bauru, Universidade de São Paulo; 2009.

20. Garone MG; Russo EMA; Netto NG. Força de união por tração de uma resina composta a três porcelanas dentais tratadas com ácido fluorídrico. Rev. odontol. Univ. Cid. São Paulo 2006; 18(1):37-42.

21. Ozcan M, Akkaya A. New approach to bonding all-ceramic adhesive fixed partial dentures: A clinical report. J Prosthet Dent 2002;88:252-254. 
22. Boscato N. Caracterização cerâmica e avaliação fractográfica da interface adesiva com resina, após diferentes tratamentos de superfície. Piracicaba. [tese]. Campinas, SP: Faculdade de Odontologia Estadual de Campinas, 2005.

23. Michida SMA, Valandro LF, Yoshiga S, Andreatta Filho OD, Balducci I, Bottino MA. Efeito do tratamento de superfície de uma cerâmica aluminizada infiltrada de vidro sobre a resistência à microtração. J Appl Oral Sci 2003;11(4):361-366.

24. Haselton DR, Diaz-Arnold AM, Dunne JT. Shear bond strengths of 2 intraoral porcelain repair systems to porcelain or metal substrates. J Prosthet Dent 2001;86(5).

25. Panah FG, Rezai MSM, Ahmadian L. The Influence of Ceramic Surface Treatments on the Micro-shear Bond Strength of Composite Resin to IPS Empress 2. J. Prosthodont. 2008;17(5): 409-414.

26. Matinlinna JP, Vallittu PK. Bonding of resin composites to etchable ceramic surfaces - an insight review of the chemical aspects on surface conditioning. J Oral Rehabil 2007;34:622-630.

27. Raigrodski AJ. Contemporary All-ceramic Fixed Partial Dentures: A review. Dent Clin N Am 2004;48:531-544.

28. Jorge MR. Rugosidade de superfície e resistência flexural do IPS Empress 2 submetida a seis tratamentos de superfície de superfície. Curitiba. [dissertação]. Curitiba: Pontifícia Universidade Católica do Paraná; 2007.

29. Aras WMF, León BLT. Tratamento de superfície e cimentação adesiva de cerâmicas aluminizadas: revisão de literatura. Rev. odontol. UNESP 2009;38(2):93-98.

30. Suttor D, Bunke K, Hoescheler S, Hauptmann H, Hertlein G. LAVA-The system for allceramic ZrO2 crown and bridge frameworks. Int J Comput Dent 2001;4(3):195-206.

31. Spohr AM, Sobrinho LC, Cansani S, Sinhoret MAC, Knowles JC. Influence of surace conditions and silane agent on the Bond of resin to IPS Empress 2 Ceramic. Int J Prosthodont 2003;16(3):277-282. 